\title{
Desenvolvimento de um Algoritmo Multi-Populacional Auto-Adaptativo para a Resolução de Problemas de Otimização
}

\section{Development of a Self-Adaptive Multi-Population Algorithm to Solve Optimization Problems}

Camila Campos Carvalho ${ }^{1}$, Fran Sérgio Lobato²

\section{RESUMO}

Nos últimos anos, inúmeras são as estratégias propostas para a adaptação dos parâmetros de entrada dos algoritmos de otimização, que são considerados constantes durante o processo de busca pelo ótimo global. No contexto evolutivo, tal característica pode levar a ótimos locais ou impossibilitar a exploração de regiões do espaço de busca. Neste sentido, o presente trabalho tem por objetivo o desenvolvimento de uma abordagem evolutiva com sub-populações resultante da associação do Algoritmo de Colônia de Vagalumes com um Modelo Caótico de Busca. Esta nova estratégia evolutiva tem como finalidade introduzir diversidade na população, bem como prevenir a convergência prematura. Os resultados obtidos com a resolução de uma série funções matemáticas e com problemas de projeto em engenharia com diferentes níveis de complexidade demonstraram a capacidade do algoritmo proposto em termos de convergência e do número de avaliações da função objetivo em comparação com o algoritmo original.

Palavras-chave: Parâmetros Auto-Adaptativos, Algoritmo de Colônia de Vagalumes, Funções Matemáticas, Projeto de Sistemas de Engenharia.

\section{ABSTRACT}

In last years, various strategies to updating the input parameters of optimization algorithms have been proposed. Traditionally, these can be considered constant during the optimization process to simplify the implementation to find the global optimum. In evolutionary context, this characteristic can lead to local optimal or impossible to explore regions of design space. In this sense, the present contribution aim the development of an evolutionary approach with sub-populations resulting from the association of the Firefly Algorithm with a Chaotic Search Model to introduce diversity in the population and to avoid premature convergence. The results obtained with the resolution of mathematical functions and engineering system design with different levels of complexity demonstrated the ability of the proposed algorithm in terms of convergence and number of objective function evaluations when compared with the canonical original algorithm.

Keywords: Self-Adaptive Parameters, Firefly Algorithm, Mathematical Functions, Engineering System Design.
1 Programa de Pós Graduação em Modelagem e Otimização,

Universidade Federal de Goiás,

Av. Dr. Lamartine P. Avelar, 1120,

St. Universitário, Bloco M -

IMTec, CEP 75704-020, Catalão,

GO, Brasil.

E-mail:

kacamilacarvalho@hotmail.com

${ }^{2}$ Faculdade de Engenharia Química, Universidade Federal

de Uberlândia, Av. João Naves de

Ávila 2121 - Campus Santa

Mônica, Bloco 1K, CEP 38408-

144, Uberlândia, MG, Brasil.

E-mail:

fslobato@ufu.br 


\section{INTRODUÇAO}

Tradicionalmente, os parâmetros de quaisquer métodos de otimização são considerados constantes durante o processo de busca pela solução global. Apesar desta característica simplificar os algoritmos, o uso de parâmetros constantes pode levar a convergência para uma solução local ou pode fazer com que regiões do espaço de projeto não sejam exploradas. No contexto evolutivo, a incorporação de operadores para a atualização de um ou mais parâmetros nestes algoritmos vem de encontro ao que se observa na natureza como, por exemplo, a variação do número de indivíduos que compõem o tamanho das espécies. Assim, por que não desenvolver algoritmos com parâmetros adaptáveis em cada geração?. Neste cenário, deixa-se de considerar, pelo menos de forma mais direta, a influência da escolha destes parâmetros por parte do usuário de determinado algoritmo.

Como um dos mais tradicionais representantes da classe de métodos de otimização bio-inspirados na natureza, pode-se citar o Algoritmo de Colônia de Vagalumes (ACV), ou simplesmente Algoritmo Vagalume (Firefly Algorithm) proposto por Yang (2008). Este tem por característica modelar a interação entre os vagalumes através da simulação da etapa de acasalamento. Desde a sua introdução no cenário de otimização, inúmeros são os trabalhos envolvendo este algoritmo na resolução de diferentes tipos de problemas, dentre os quais pode-se citar: a sincronização de sensores em rede (WERNER-ALLEN et al., 2005); o projeto de estabilizadores de sistemas de força (DAS e VENAYAGAMOORTHY, 2006); o projeto de redes sem fio (LEIDENFROST e ELMENREICH, 2008); a otimização de funções matemáticas (YANG, 2008); a resolução de um problema inverso de condução de calor (LUZ et al., 2009); a aplicação a problemas de programação discretos (SAYADI et al., 2010); a resolução de problemas de projeto multimodais altamente não-lineares (AZAD e AZAD, 2011); o projeto de uma antena (BASU e MAHANTI, 2011; CHATTERJEE et al., 2012), um estudo comparativo entre o ACV com outros algoritmos de otimização para a resolução de problemas de clustering (SENTHILNATH el al., 2011); a otimização em ambientes dinâmicos (FARAHANI et al. (2011a,b); ABSHOURI et al.; 2011); na compressão de imagens digitais (HORNG et al., 2012); dentre outras aplicações.

Nos últimos anos, inúmeras modificações no ACV podem ser encontradas na literatura especializada na tentativa de aumentar o desempenho do mesmo. Aydilek (2018) propôs um algoritmo híbrido que combina otimização de vagalumes e enxame de 
partículas para a resolução de problemas numéricos. Wang et al. (2018) propuseram uma nova variante do ACV que apresenta um operador denominado de atração de vizinhança em que cada vagalume é atraído por outros mais brilhantes selecionados a partir de uma vizinhança predefinida, em vez daqueles de toda a população. O algoritmo proposto é testado em uma série de funções matemáticas. Lieu et al. (2018) apresentaram um algoritmo híbrido que envolve o $\mathrm{ACV}$ e o Algoritmo de Evolução Diferencial. Nesta estratégia, um parâmetro utilizado para selecionar o esquema de mutação e que pondera as buscas global e local é automaticamente adaptado. A metodologia proposta foi empregada para otimização de forma e tamanho de estruturas de treliça sob restrições de múltiplas frequências.

Diante do que foi apresentado, o objetivo deste trabalho é propor uma nova metodologia para a atualização dinâmica dos parâmetros do ACV considerando: (I) a introdução de uma estratégia para atualização do tamanho da população; (ii) uma estratégia baseada em um Modelo Caótico de Busca para a atualização do fator de atratividade e do parâmetro de absorção da luz pelo meio; (iii) uma relação empírica para a atualização do parâmetro de inserção de regularidade; e (iv) o conceito de subpopulações para a inserção de diversidade ao processo de busca pela solução global. Este trabalho está estruturado como segue: a seção 2 apresenta as características gerais do ACV; já na seção 3 é apresentada a descrição da metodologia proposta neste trabalho. Na seção 4 é apresentada os resultados obtidos com a aplicação da metodologia proposta. Finalmente, as conclusões são apresentadas na última seção.

\section{ALGORITMO DE COLÓNIA DE VAGALUMES}

O ACV é conceitualmente baseado na característica bio-luminescente de vagalumes, insetos coleópteros notórios por suas emissões luminosas. Dentre as funções desempenhadas por esta luminescência pode-se enumerar (YANG, 2008): (I) ferramenta de comunicação e atração para potenciais parceiros na reprodução; (ii) isca para atração de eventuais presas para o vagalume; (iii) mecanismo de alerta para potenciais predadores.

A função da comunicação através dessa bioluminescência advém do fato observado de que, em determinadas espécies de vagalumes, a taxa de intermitência e a intensidade dos flashes luminosos é parte essencial do mecanismo que os vagalumes utilizam para o ritual de acasalamento (as fêmeas são atraídas pelo brilho emitido pelos machos). Além disso, quando existe uma grande quantidade destes em uma área comum, o fenômeno de 
sincronização é responsável pela emissão dos flashes luminosos, evidenciando uma característica de auto-organização emergente (YANG, 2008).

Fundamentado nesta ideia, Yang (2008) propôs o ACV considerando as seguintes hipóteses: (I) os vagalumes não possuem sexo, portanto qualquer vagalume poderá atrair ou ser atraído; (ii) a atratividade é proporcional ao brilho emitido e decai conforme aumenta a distância entre os vagalumes (regra baseada em observação do comportamento animal); (iii) o brilho emitido por um vagalume é determinado pela sua avaliação frente ao objetivo (i.e., quanto melhor avaliado, mais brilhante); (iv) a atratividade de um vagalume é determinada pela intensidade da luz emitida; e ( $v$ ) a determinação da intensidade emitida é função de sua avaliação.

Baseado nestas hipóteses, a intensidade de emissão de luz de um vagalume é proporcional à função objetivo, i.e., $I(x) \approx J(x)$, que decai em função da distância entre os vagalumes devido à absorção da luz pelo meio. Assim, a intensidade percebida por um vagalume é dada por $I(r)=I_{0} \exp \left(\gamma r^{2}\right)$, em que $I_{0}$ é a intensidade da luz emitida; $r$ é a distância Euclidiana entre os vagalumes $i$ e $j$, sendo $i$ o vagalume mais brilhante e $j o$ vagalume menos brilhante; e $\gamma$ é o parâmetro de absorção da luz pelo meio. A atratividade $\beta$, responsável pela aproximação dos vagalumes, é definida como:

$$
\beta=\beta_{0} \exp \left(-\gamma r^{2}\right)
$$

em que $\beta_{0}$ é o fator de atratividade para uma distância $r=0$. A movimentação em um dado passo de tempo $t$ do vagalume $i$ em direção a um melhor vagalume $j$, em termos da função objetivo, é definida como:

$$
x_{i}^{t}=x_{i}^{t-1}+\beta\left(x_{j}^{t-1}-x_{i}^{t-1}\right)+\alpha(\text { rand }-0,5)
$$

$\mathrm{Na}$ equação acima, o segundo termo do lado direito insere a atratividade $\beta$, enquanto o terceiro termo, ponderado pelo parâmetro $\alpha$, regula a inserção de certa aleatoriedade no caminho percorrido pelo vagalume, onde rand é um número aleatório entre 0 e 1.

A Figura 1 apresenta o pseudocódigo do ACV.

No ACV, o usuário deve informar os seguintes parâmetros: o número de vagalumes $(N P)$, o número de variáveis de projeto $(D)$, o fator de atratividade $\left(\beta_{0}\right)$, o parâmetro de absorção da luz pelo meio $(\gamma)$, o parâmetro de inserção de regularidade $(\alpha)$, e o número máximo de gerações $\left(N_{g e n}\right)$. 


\section{Algoritmo de Colônia de Vagalumes}

Início

Definir a função objetivo $J(x), x=\left(x_{1}, x_{2}, \ldots, x_{D}\right)^{\top}$

Definir os parâmetros do ACV

Para $i=1$ até o número máximo de gerações faça

Calcular a intensidade da luz $l_{i}$ para $x_{i}$ proporcionalmente a $J\left(x_{i}\right)$

Para $j=1$ até o número de vagalumes

Calcular o fator de atratividade

Mover o vagalume jem direção aos vagalumes mais brilhantes

Verificar se o vagalume está dentro dos limites

Fim-Para

Fim-Para

Pós-processamento e visualização dos resultados

Fim

Figura 1. Pseudocódigo para o ACV.

\section{METODOLOGIA}

A presente seção tem como objetivo apresentar os operadores para a atualização dos parâmetros do ACV, bem como a estratégia de sub-população considerada.

\subsection{Atualização do Tamanho da População}

Para propor uma relação para a atualização do tamanho da população no ACV, será considerado um novo parâmetro, a saber, a taxa de convergência ( $T C)$, definido como sendo:

$$
T C=\frac{f_{\text {average }}}{f_{\text {worst }}}
$$

em que $f_{\text {average }}$ e $f_{\text {worst }}$ representam os valores médio e pior em termos da função objetivo obtida em cada geração, respectivamente.

A TC pode ser interpretada como a forma pela qual a homogeneidade da população no processo evolutivo pode ser mensurada. Inicialmente tem-se TC próximo de zero, isto é, o "pior" valor da função objetivo é bem diferente do valor médio da função objetivo, o que faz com que a taxa seja próxima a zero. Por outro lado, com a evolução do processo de otimização, como a população tende a ficar homogênea, TC tende a 1. Assim, a partir 
desta definição pode-se propor a seguinte relação para a atualização do número de indivíduos na próxima geração:

$$
N P=\operatorname{round}\left(N P_{\min } T C+N P_{\max }(1-T C)\right)
$$

em que $N P_{\text {min }}$ e $N P_{\text {max }}$ representam os valores mínimo e máximo para o tamanho da população, respectivamente, e o operador round(.) indica o arredondamento para o inteiro mais próximo. Neste caso, através desta equação o número de indivíduos da população é atualizado através do uso de informações durante a evolução do processo em análise, isto é, se a população tende a se tornar homogênea, a população tende ao seu valor mínimo. Por outro lado, se a população não tende a se tornar homogênea, a população tende ao seu valor máximo.

Em termos práticos, durante a utilização desta estratégia devem-se considerar três possibilidades:

- Se o tamanho da nova população $\left(N P_{\text {new }}\right)$ não se altera com relação ao tamanho atual $\left(N P_{\text {old }}\right)$, isto é, $N P_{\text {new }}=N P_{\text {old }}$, nenhuma providência é tomada;

- Se o tamanho da população na próxima geração diminui com relação à geração corrente $\left(N P_{\text {new }}<N P_{\text {old }}\right)$, ordena-se os indivíduos da população corrente de acordo com a função objetivo e somente os $N P_{\text {new }}$ indivíduos participarão da nova população;

- Se o tamanho da população na próxima geração aumenta com relação à geração corrente $\left(N P_{\text {new }}>N P_{\text {old }}\right)$, gera-se $N P_{\text {new }} N P_{\text {old }}$ indivíduos através do operador para a geração de indivíduos no ACV, e esses são acrescentados à população atual para formar a nova população com $N P_{\text {new }}$ indivíduos.

Com o operador proposto, o número de indivíduos da população pode ser atualizado dinamicamente durante o processo de otimização. Cabe ressaltar que o número de indivíduos da população pode aumentar ou diminuir de acordo com a homogeneidade da população. Além disso, se a população não se tornar homogênea, este operador tende a trabalhar com a população máxima definida pelo usuário, conforme acontece no ACV proposto por Yang (2008).

\subsection{Atualização do Fator de Atratividade e do Parâmetro de Absorção de Luz}

Para a atualização do fator de atratividade $\left(\beta_{0}\right)$ e do parâmetro de absorção da luz pelo meio $(\gamma)$ será considerado um Modelo Caótico de Busca. A escolha por este tipo de abordagem para a atualização destes parâmetros se deve à diversidade introduzida 
através do conceito de caos. Segundo Tavazoei e Haeri (2007), o caos pode ser entendido como um gerador randômico obtido através de sistemas determinísticos. Segundo Li e Jiang (1998), nos algoritmos de otimização com busca caótica, a convergência prematura pode ser prevenida através do movimento caótico incorporado pelos modelos. Segundo Yang et al. (2007), a ideia básica de um algoritmo de otimização fundamentado em um modelo caótico de busca consiste primeiramente na definição de um gerador de caos baseado em alguma relação matemática (conhecida como mapa caótico). De posse desse gerador, uma sequência de "pontos caóticos" dentro da faixa considerada como interessante para cada um dos parâmetros que se deseja atualizar.

Neste contexto, para fins de atualização dos parâmetros do ACV citados, será utilizado o modelo de Mapa Logístico para essa finalidade. Esse é definido pela seguinte relação (DEVANEY, 1987; OTT, 2002):

$$
x_{i+1}=\mu x_{i}\left(1-x_{i}\right)
$$

em que $x$ é $o$ parâmetro que se deseja atualizar e $\mu$ é uma constante que define 0 comportamento caótico para este modelo $(3,57 \leq \mu \leq 4)$. Assim, conhecendo-se um ponto de partida (parâmetro inicial pertencente a faixa definida como interessante pela literatura), pode-se atualizar o fator de atratividade $\left(\beta_{0}\right)$ e o parâmetro de absorção da luz pelo meio $(\gamma)$ da seguinte forma:

$$
\begin{aligned}
& \beta_{0}^{k+1}=\mu \beta_{0}^{k}\left(1-\beta_{0}^{k}\right) \\
& \gamma^{k+1}=\mu \gamma^{k}\left(1-\gamma^{k}\right)
\end{aligned}
$$

em que $\beta_{0}^{k+1}$ e $\gamma^{k+1}$ são os valores atualizados desses parâmetros na geração $k+1$ a partir do valor default encontrado na literatura. Dessa forma, esses parâmetros são atualizados a cada geração segundo um modelo que introduz diversidade a esses parâmetros e, por consequência, ao processo evolutivo.

\subsection{Parâmetro de Inserção de Regularidade}

Já a atualização do parâmetro de inserção de regularidade $(\alpha)$ pode ser realizada a partir da definição de uma relação empírica, baseada no trabalho de Yang (2008), onde este parâmetro é inicializado como um valor próximo à unidade (para aumentar a diversidade da população no início do processo evolutivo) e com o passar do processo de otimização o mesmo é reduzido a um valor próximo a zero (para promover o refinamento 
da solução ótima corrente). Neste contexto, o presente trabalho define a seguinte relação para atualizar este parâmetro:

$$
\alpha^{k+1}=0,9 \exp (-0,05 k)
$$

em que $k+1$ é a geração corrente e 0,9 e 0,05 são parâmetros definidos a partir do estudo proposto por Yang (2008). Nesta relação percebe-se que o valor 0,9 vai sendo reduzido ao logo do processo evolutivo.

\subsection{Sub-Populações}

A definição de TC proposto anteriormente permite a atualização do tamanho da população ao longo do processo evolutivo. Neste caso, como em todo e qualquer processo evolutivo, espera-se que ao longo do mesmo a população se torne homogênea e, consequentemente, a TC se aproxime da unidade e o tamanho da população alcance o seu valor mínimo. Embora este processo seja interessante do ponto de vista da redução do número de avaliações da função objetivo, o mesmo pode convergir para uma solução local quando o tamanho da população reduz drasticamente. Esta é a razão pela qual a grande maioria dos algoritmos evolutivos inicia-se o processo com uma população de tamanho razoável.

Neste cenário, para reduzir a chance do algoritmo proposto fica estagnado em torno de um ótimo local, será considerado o uso de sub-populações para inserir diversidade ao processo. Neste caso, por exemplo, definindo-se duas populações, uma será atualizada considerando a estratégia clássica proposta por Yang (2008) e a outra será atualizada segundo um outro mecanismo evolutivo. Assim, considere como alternativa para a geração da população a seguinte relação baseada no Algoritmo de Enxame de Partículas (KENNEDY e EBERHART, 1995):

$$
x_{i}^{k+1}=x_{a}^{k}+\beta_{0}\left(x_{b}^{k}-x_{c}^{k}\right)
$$

em que os subscritos $a, b$ e $c$ representam posições de três vagalumes na população corrente (escolhido aleatoriamente dentro da população) e $\beta_{0}$ é o fator de atratividade apresentado anteriormente.

Neste caso, definindo um percentual para cada sub-população, as mesmas serão atualizadas considerando abordagens diferentes, a saber, uma considerando o mecanismo proposto no ACV e a outra considerando essa nova proposta baseada no Algoritmo de Enxame de Partículas. Cabe ressaltar que a divisão da população nestas 
duas sub-populações ocorre de forma aleatória, o que ajuda a promover a diversidade da população como um todo, já que existe a transferência de informações entre as duas subpopulações a cada geração.

\section{RESULTADOS E DISCUSSÃO}

\subsection{Funções Matemáticas}

Com o intuito de comparar o desempenho da estratégia proposta neste trabalho e denominada de ACVA (Algoritmo de Colônia de Vagalumes Adaptativo), as seguintes funções matemáticas que devem ser minimizadas são consideradas (ADJIMAN et al., 1998; ALI e TORN, 2004; CSENDES e RATZ, 1997; RAHNAMYAN et al., 2007a,b; SARIMVEIS e NIKOLAKOPOULOS, 2005; WANG et al., 2007; YANG, 2010):

1. Função $F_{1}$

$$
f(x)=x_{1}^{2}+x_{2}^{2}-\cos \left(18 x_{1}\right)-\cos \left(18 x_{2}\right)
$$

com $-1 \leq x_{i} \leq 1(i=1,2)$. Essa função apresenta cerca de 50 mínimos locais e um mínimo global igual a $-2\left(x_{i}\right.$ iguais a $\left.0, i=1,2\right)$.

2. Função $F_{2}$

$$
f(x)=x_{1}^{2}+x_{2}^{2}-0,3 \cos \left(3 \pi x_{1}\right)-0,4 \cos \left(4 \pi x_{2}\right)+0,7
$$

com $-100 \leq x_{i} \leq 100(j=1,2)$. Essa função apresenta inúmeros mínimos locais e o mínimo global é igual a $0\left(x_{i=0}, i=1,2\right)$.

3. Função $F_{3}$

$$
f(x)=\sum_{j=1}^{n}\left(100\left(x_{j}^{2}-x_{j+1}\right)^{2}+\left(x_{j}-1\right)^{2}\right)
$$

com $-5 \leq x_{i} \leq 5(i=1,2, \ldots, n=5)$. A função $F_{3}$ tem inúmeros mínimos locais e um ótimo global igual a $0\left(x_{i}=1, i=1, . ., n=5\right)$.

4. Função $F_{4}$

$$
f(x)=\sum_{j=1}^{n}\left|x_{i}\right|+\prod_{j=1}^{n}\left|x_{i}\right|
$$


com $-100 \leq x_{i} \leq 100(i=1,2, \ldots, n=20)$. A função $F_{4}$ tem ótimo global igual a $0\left(x_{i}=0, i=1, \ldots\right.$, $n=20)$.

5. Função $F_{5}$

$$
f(x)=\cos \left(x_{1}\right) \sin \left(x_{2}\right)-x_{1} /\left(x_{2}^{2}+1\right)
$$

com $0 \leq x_{i} \leq 10(\mathrm{i}=1,2)$. A função $F_{5}$ tem mínimo global igual a $-2.02181\left(x_{1}=2\right.$ e $x_{2}$ $=0,10578)$.

6. Função $F_{6}$

$$
f(x)=0,6+\sum_{i=1}^{2}\left(\sin \left(\frac{16}{15} x_{i}-1\right)+\sin ^{2}\left(\frac{16}{15} x_{i}-1\right)+\frac{1}{50} \sin \left(4\left(\frac{16}{15} x_{i}-1\right)\right)\right)
$$

com $-1 \leq x_{i} \leq 1(i=1,2)$. A função $F_{6}$ tem mínimo global igual a $0,060447\left(x_{1}=0,45834282\right.$ e $\left.x_{2}=0,45834282\right)$.

7. Função $F_{7}$

$$
f(x)=\sum_{i=1}^{n}\left|x_{i} \sin \left(x_{i}\right)+0,1 x_{i}\right|
$$

com $-10 \leq x_{i} \leq 10$ e mínimo igual a $0\left(x_{i}=0, i=1, \ldots, n=10\right)$.

8. Função $F_{8}$

$$
f(x)=\sum_{i=1}^{n} x_{i}^{6}\left(2+\sin \left(\frac{1}{x_{i}}\right)\right)
$$

com $-1 \leq x_{i} \leq 1$ e mínimo igual a $0\left(x_{i}=0, i=1, \ldots, n=10\right)$.

9. Função $F_{9}$

$$
f(x)=-\exp \left(-0,5 \sum_{i=1}^{n} x_{i}^{2}\right)
$$

com $-1 \leq x_{i} \leq 1(i=1,2, \ldots, n=10)$ e mínimo igual a $-1\left(x_{i}=0, i=1, \ldots, n=10\right)$.

10. Função $F_{10}$

$$
f(x)=\exp \left(-\sum_{i=1}^{n}\left(\frac{x_{i}}{\lambda}\right)^{2 m}\right)-2 \exp \left(-\sum_{i=1}^{n}\left(x_{i}-\pi\right)^{2}\right) \prod_{i=1}^{n} \cos ^{2}\left(x_{i}\right)
$$


com $-10 \leq x_{i} \leq 10(i=1,2, \ldots, n=10)$ e mínimo global considerando $m=5$ e $\lambda=15$ igual a $-1\left(x_{i}\right.$ $=\pi, i=1,2, \ldots, n=10)$.

Para fins de aplicação, alguns pontos devem ser destacados:

- O desempenho do ACVA será comparado considerando o ACV com três diferentes configurações: $\operatorname{ACV}^{1}\left(N P=100, N_{g e n}=2000, \alpha=0,9 ; \beta_{0}=0,8\right.$ e $\left.\gamma=1\right) ; A^{2} V^{2}(N P=100$, $N_{\text {gen }}=2000, \alpha=0,8 ; \beta_{0}=0,9$ e $\left.\gamma=0,9\right)$ e $A_{C V}^{3}\left(N P=100, N_{g e n}=2000, \alpha=0,7 ; \beta_{0}=0,8\right.$ e $V=0,9)$;

- Para a metodologia proposta foram considerados fixos os seguintes parâmetros: $N P_{\min }=20, N P_{\max }=100, N_{g e n}=2000$, e os parâmetros $\alpha, \beta_{0}$ e $\gamma$ sendo atualizados conforme descrito anteriormente;

- Para a obtenção dos valores médios que serão apresentados a seguir, todos os casos foram resolvidos 10 vezes considerando diferentes sementes iniciais para a inicialização do gerador de números aleatórios;

- Para finalizar o processo iterativo em cada algoritmo será considerado a homogeneidade da população, isto é; quando a diferença (em módulo) entre o valor médio e o pior valor referente a função objetivo for menor que $10^{-6}$, o processo de otimização é finalizado. Caso a população não se torne homogênea, o processo continua até que o número de gerações seja igual a $N_{g e n}$;

- Para mensurar a redução obtida (com relação ao número de avaliações da função objetivo do ACV com $N_{\text {gen }}$ ), definiu-se o parâmetro $\Gamma$ como sendo a taxa de redução:

$$
\Gamma=100 \%-\left(\frac{\Phi}{N P \times N_{g e n}+N P}\right) 100 \%
$$

Cabe ressaltar que este parâmetro representa a redução percentual que se obtêm com a aplicação do algoritmo $\Phi\left(\Phi=\mathrm{ACV}^{1}, \mathrm{ACV}^{2}, \mathrm{ACV}^{3}\right.$, ou $\left.\mathrm{ACVA}\right)$ em relação ao algoritmo que é finalizado considerando um número fixo de gerações.

$\mathrm{Na}$ Tabela 1 são apresentados os melhores resultados, os piores e os valores médios em relação à cada função objetivo, o número médio de avaliações da função objetivo (NAFO) e a redução percentual $(\Gamma)$. 
DOI 10.18605/2175-7275/cereus.v10n2p252-275
CARVALHO, C. C.; LOBATO, F. S.

Desenvolvimento de um Algoritmo Multi-Populacional Auto-Adaptativo para a Resolução de Problemas de Otimização

Tabela 1. Resultados obtidos pelas estratégias $\mathrm{ACV}^{1}, \mathrm{ACV}^{2}, \mathrm{ACV}^{3}$ e $\mathrm{ACVA}$.

\begin{tabular}{|c|c|c|c|c|c|}
\hline & & $\overline{\mathrm{ACV}^{1}}$ & $\mathrm{ACV}^{2}$ & $\mathrm{ACV}^{3}$ & $\overline{A C V A}$ \\
\hline \multirow{3}{*}{$F_{1}$} & Worst & $-2,0000$ & $-1,9999$ & $-2,0000$ & $-2,0000$ \\
\hline & Average & $-2,0000$ & $-2,0000$ & $-2,0000$ & $-2,0000$ \\
\hline & Best & $-2,0000$ & $-2,0000$ & $-2,0000$ & $-2,0000$ \\
\hline NAFO & & 173740 & 164080 & 158260 & 22339 \\
\hline$\Gamma(\%)$ & & 13,17 & 18,01 & 20,90 & 88,83 \\
\hline \multirow{3}{*}{$F_{2}$} & Worst & $1,1343 \mathrm{E}-07$ & 1,8771E-07 & $1,3885 \mathrm{E}-07$ & $1,5455 \mathrm{E}-06$ \\
\hline & Average & $1,2574 \mathrm{E}-07$ & $4,1999 \mathrm{E}-08$ & $4,2555 \mathrm{E}-08$ & $6,5556 \mathrm{E}-07$ \\
\hline & Best & 1,3552E-08 & $2,5998 \mathrm{E}-09$ & 2,2227E-09 & $3,8776 \mathrm{E}-12$ \\
\hline NAFO & & 137100 & 145920 & 186240 & 100092 \\
\hline$\Gamma(\%)$ & & 31,48 & 27,07 & 6,92 & 49,97 \\
\hline \multirow{3}{*}{$F_{3}$} & Worst & 2,8777E-02 & 5,6666E-02 & 6,5716E-02 & 3,3433E-04 \\
\hline & Average & 2,3322E-02 & $3,4545 \mathrm{E}-02$ & 5,7771E-02 & $2,7444 \mathrm{E}-05$ \\
\hline & Best & $3,0338 \mathrm{E}-03$ & $1,1664 \mathrm{E}-02$ & 4,7770E-03 & $1,4331 \mathrm{E}-07$ \\
\hline NAFO & & 200100 & 200100 & 140700 & 79238 \\
\hline$\Gamma(\%)$ & & - & - & 29,68 & 60,40 \\
\hline \multirow{3}{*}{$F_{4}$} & Worst & 2,8876E-03 & $2,3999 \mathrm{E}-03$ & 2,9994E-03 & $1,2254 \mathrm{E}-02$ \\
\hline & Average & $2,5876 \mathrm{E}-03$ & $2,1666 \mathrm{E}-03$ & 1,1991E-03 & 4,7555E-03 \\
\hline & Best & 1,9996E-03 & $1,4544 \mathrm{E}-03$ & 1,6807E-03 & 3,5543E-05 \\
\hline NAFO & & 200100 & 200100 & 200100 & 90779 \\
\hline$\Gamma(\%)$ & & - & - & - & 54,63 \\
\hline \multirow{3}{*}{$F_{5}$} & Worst & $-2,0218$ & $-2,0218$ & $-2,0218$ & $-2,0218$ \\
\hline & Average & $-2,0218$ & $-2,0218$ & $-2,0218$ & $-2,0218$ \\
\hline & Best & $-2,0218$ & $-2,0218$ & $-2,0218$ & $-2,0218$ \\
\hline NAFO & & 200100 & 200100 & 200100 & 19294 \\
\hline$\Gamma(\%)$ & & - & - & - & 90,36 \\
\hline \multirow{3}{*}{$F_{6}$} & Worst & $6,4470 \mathrm{E}-02$ & 6,4470E-02 & $6,4470 \mathrm{E}-02$ & $6,4470 \mathrm{E}-02$ \\
\hline & Average & $6,4470 \mathrm{E}-02$ & $6,4470 \mathrm{E}-02$ & $6,4470 \mathrm{E}-02$ & $6,4470 \mathrm{E}-02$ \\
\hline & Best & $6,4460 \mathrm{E}-02$ & $6,4470 \mathrm{E}-02$ & $6,4470 \mathrm{E}-02$ & $6,4470 \mathrm{E}-02$ \\
\hline NAFO & & 200100 & 200100 & 200100 & 21937 \\
\hline$\Gamma(\%)$ & & 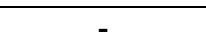 & - & - & 89,03 \\
\hline \multirow{3}{*}{$F_{7}$} & Worst & 7,6994E-05 & 2,2453E-05 & 6,8999E-05 & $1,3333 \mathrm{E}-06$ \\
\hline & Average & 6,8444E-06 & $4,2444 \mathrm{E}-06$ & $3,9444 \mathrm{E}-06$ & $3,6443 \mathrm{E}-07$ \\
\hline & Best & 0 & 0 & 0 & 0 \\
\hline NAFO & & 80240 & 49740 & 75100 & 28850 \\
\hline$\Gamma(\%)$ & & 59,90 & 75,14 & 62,46 & 85,58 \\
\hline \multirow{3}{*}{$F_{8}$} & Worst & 0 & 0 & 0 & 0 \\
\hline & Average & 0 & 0 & 0 & 0 \\
\hline & Best & 0 & 0 & 0 & 0 \\
\hline NAFO & & 23680 & 27280 & 24440 & 16380 \\
\hline$\Gamma(\%)$ & & 88,16 & 86,36 & 87,78 & 91,32 \\
\hline \multirow{3}{*}{$F_{9}$} & Worst & -1 & -1 & -1 & -1 \\
\hline & Average & -1 & -1 & -1 & -1 \\
\hline & Best & -1 & -1 & -1 & -1 \\
\hline NAFO & & 42640 & 50920 & 64180 & 22084 \\
\hline$\Gamma(\%)$ & & 78,69 & 74,55 & 67,92 & 88,96 \\
\hline \multirow{3}{*}{$F_{10}$} & Worst & $-9,9992 \mathrm{E}-01$ & $-9,9998 \mathrm{E}-01$ & $-9,9997 \mathrm{E}-01$ & $-9,9999 \mathrm{E}-01$ \\
\hline & Average & $-9,9997 \mathrm{E}-01$ & $-9,9998 \mathrm{E}-01$ & $-9,9998 \mathrm{E}-01$ & $-9,9999 \mathrm{E}-01$ \\
\hline & Best & $-9,9999 \mathrm{E}-01$ & $-9,9999 \mathrm{E}-01$ & $-9,9999 \mathrm{E}-01$ & $-9,9999 \mathrm{E}-01$ \\
\hline NAFO & & 200100 & 200100 & 200100 & 20074 \\
\hline$\Gamma(\%)$ & & - & - & - & 89,97 \\
\hline
\end{tabular}

Nesta tabela percebe-se que, primeiramente, todas as versões do ACV utilizadas convergiram para a solução ótima dos problemas considerados. Em segundo lugar, que a 
escolha dos parâmetros utilizados no ACV influencia, como esperado, o número total de avaliações da função objetivo requeridas para encontrar a solução. Para muitos estudos de caso, quando os parâmetros empregados pelo ACV são considerados constantes, a população não se torna homogênea e o processo evolutivo encerra-se quando o valor máximo de gerações é alcançado. Neste caso o valor máximo de avaliações da função objetivo é alcançado (200100).

Em termos de convergência, a metodologia proposta sempre convergiu para a solução ótima. Todavia, como observado na Tabela 1, o custo computacional (NAFO) sempre foi menor que aqueles requeridos quando os parâmetros foram considerados constantes. Isto se deve ao fato dos parâmetros serem atualizados dinamicamente, favorecendo a obtenção da solução ótima, bem como a homogeneidade da população durante o processo evolutivo, fazendo com que o número de indivíduos alcançasse o seu valor mínimo. Assim, o valor do parâmetro $\Gamma$, que representa o ganho percentual em se reduzir o tamanho da população ao longo do processo de otimização, sempre foi maior do que os requeridos (observa-se uma redução da ordem de aproximadamente 50 à $90 \%$ do custo total requerido para a resolução das funções matemáticas em relação aos algoritmos $A C V^{1}, A C V^{2}$ e $\left.A C V^{3}\right)$. Isto significa que pode-se resolver um mesmo problema com um esforço computacional muito menor do que quando considera-se os parâmetros fixos durante o processo evolutivo.

A Figura 2 apresenta o valor da função objetivo - FO (melhor, pior e médio), da TC, de $N P$ e dos parâmetros adaptados ao longo do processo evolutivo para a função $F_{1}$.

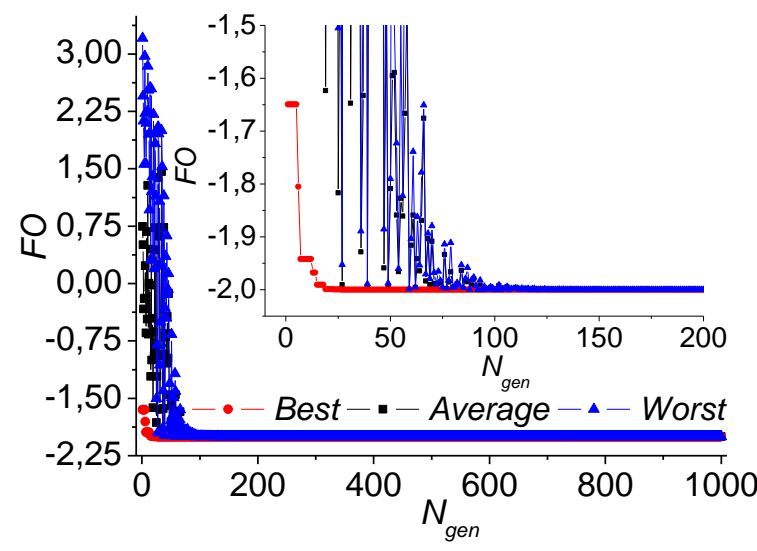

(a) Função Objetivo.

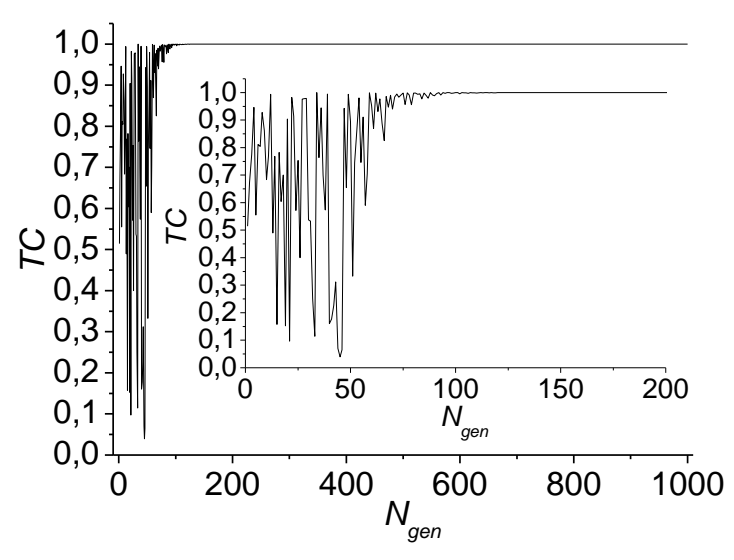

(b) Taxa de Convergência. 


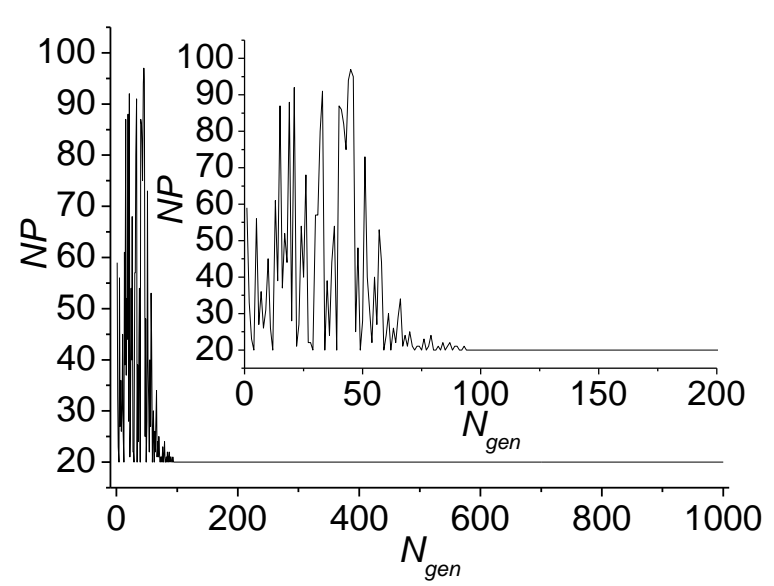

(c) Tamanho da População.

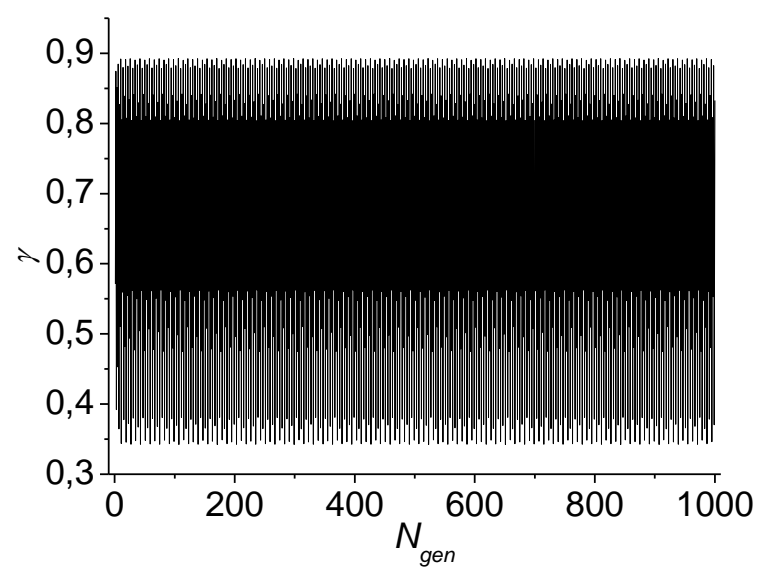

(e) Parâmetro de absorção da luz.

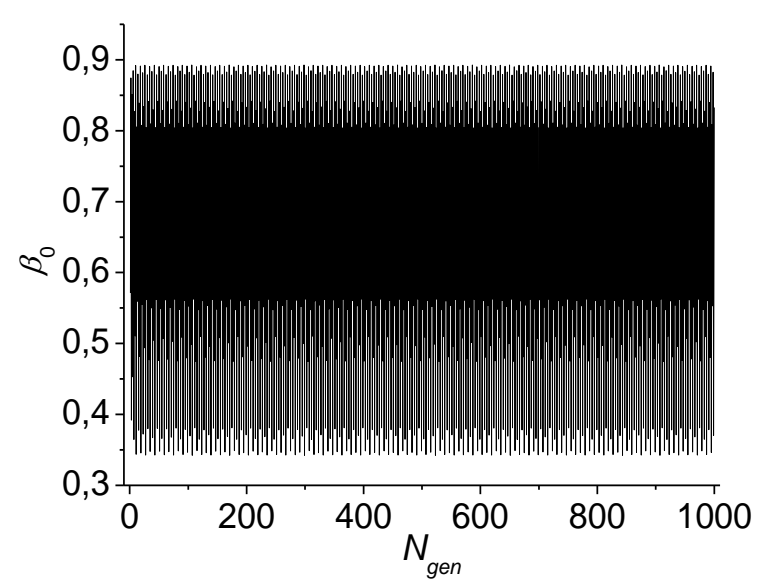

(d) Fator de atratividade.

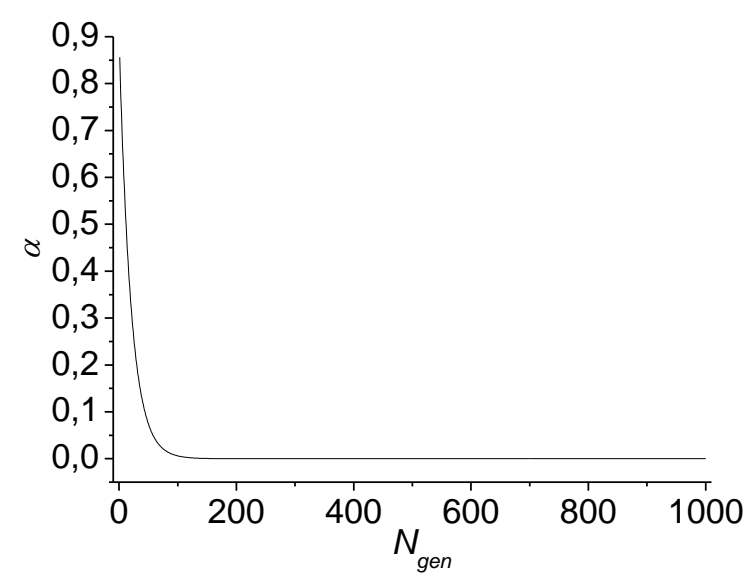

(f) Parâmetro de Inserção de Regularidade.

Figura 2. Evolução da função objetivo e dos parâmetros do ACVA para a função $F_{1}$.

Na Figura 2(a) é possível observar que população se torna homogênea ao longo do processo evolutivo, o que implica que não é necessário continuar avaliando uma população em que os candidatos apresentam as mesmas características. Nas Figuras 2(b) e 2(c) fica claro que quando o valor de $T C$ se torna próximo à unidade, que o valor de NP tende ao seu valor mínimo. Todavia, observa-se que o valor de TC pode flutuar fazendo com que o valor de NP também flutue. Já nas Figuras 2(d) e 2(e) verifica-se a variação dos parâmetros $\beta_{0}$ e $y$ ao longo do processo evolutivo via aplicação do modelo caótico de busca considerado. Nestas figuras, ambos os parâmetros flutuam em torno dos valores que são comumente sugeridos pela literatura especializada. Finalmente, na Figura 2(f) é apresentada a variação do parâmetro $\alpha$ ao longo do processo evolutivo. Conforme descrito anteriormente, a variação deste parâmetro é realizada através de uma relação 
empírica na qual enfatiza-se inicialmente uma busca global e ao final do procedimento evolutivo, tem-se a ênfase em uma busca local.

\subsection{Projeto de Sistemas de Engenharia}

O primeiro estudo de caso de engenharia consiste do projeto de um recipiente de pressão cujo objetivo é minimizar o custo total conforme a Figura 3 (COELLO, 2000; DEB, 1997). Existem quatro variáveis no projeto: $x_{1}$ ( $T_{s}$, espessura do invólucro), $x_{2}\left(T_{h}\right.$, espessura da tampa), $x_{3}\left(R\right.$, raio interno) e $x_{4}(L$, comprimento da secção cilíndrica do recipiente), $R$ e $L$ são contínuos.

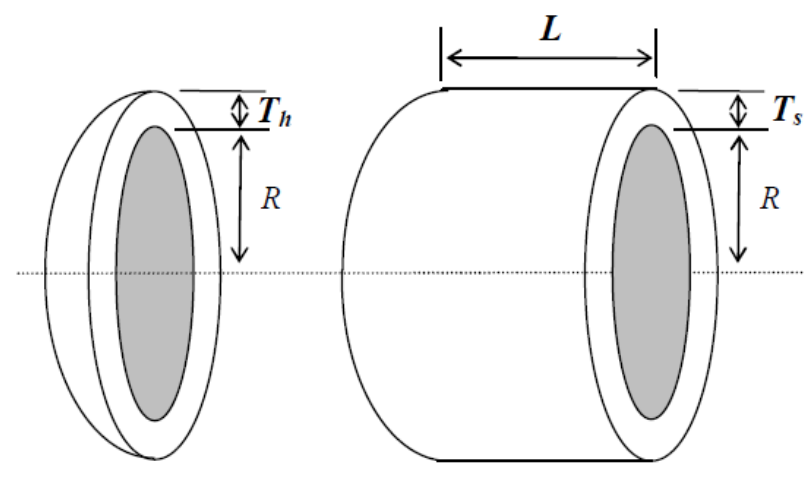

Figura 3. Projeto de um recipiente de pressão.

O problema consiste da minimização do custo associado ao projeto deste recipiente, descrito matematicamente por:

$$
\min f=0,6224 x_{1} x_{3} x_{4}+1,7781 x_{2} x_{3}^{2}+3,1661 x_{1}^{2} x_{4}+19,84 x_{1}^{2} x_{3}
$$

sujeito a restrições geométricas, limites das variáveis de projeto e de volume:

$$
\begin{gathered}
g_{1} \equiv-x_{1}+0,0193 x_{3} \leq 0 \\
g_{2} \equiv-x_{2}+0,00954 x_{3} \leq 0 \\
g_{3} \equiv-\pi x_{3}^{2} x_{4}-4 / 3 \pi x_{3}^{3}+1296000 \leq 0 \\
g_{4} \equiv x_{4}-240 \leq 0 \\
0 \leq x_{1} \leq 1, \quad 0 \leq x_{2} \leq 1, \quad 10 \leq x_{3} \leq 200, \quad 10 \leq x_{4} \leq 240
\end{gathered}
$$

Com o intuito de se comparar o desempenho da estratégia proposta neste trabalho para a resolução deste estudo de caso, considera-se o ACV (NP=100, $N_{g e n}=1000, \alpha=0,9$; $\beta_{0}=0,8$ e $\left.\gamma=0,8\right)$; ACVA $\left(N P_{\min }=20, N P_{\max }=100, N_{g e n}=1000\right.$, e os parâmetros $\alpha, \beta_{0}$ e $\gamma$ sendo atualizados conforme descrito anteriormente). Para o tratamento das restrições de 
DOI 10.18605/2175-7275/cereus.v10n2p252-275
CARVALHO, C. C.; LOBATO, F. S.

Desenvolvimento de um Algoritmo Multi-Populacional Auto-Adaptativo para a Resolução de Problemas de Otimização

desigualdade será utilizado o Método da Penalização Estática com parâmetro de penalidade da ordem de $10^{8}$ (MASTA et al., 2012).

A Tabela 3 apresenta os resultados obtidos pelos algoritmos ACV e ACVA em comparação com os obtidos por Coello (2000) e Deb (1997) usando Algoritmos Genéticos, por Hu et al. (2003) via Enxame de Partículas e por Masta et al. (2012) usando o Algoritmo Line-up.

Tabela 2. Resultados obtidos com os algoritmos ACV e ACVA.

\begin{tabular}{ccccccc}
\hline & Hu et al. (2003) & Coello (2000) & Deb (1997) & Masta et al. (2012) & ACV & ACVA \\
\hline$x_{1}\left(T_{s}\right)$ & 0,8125 & 0,8125 & 0,9375 & 0,7810 & 0,7395 & 0,7275 \\
\hline$x_{2}\left(T_{h}\right)$ & 0,4375 & 0,4375 & 0,5000 & 0,3860 & 0,3661 & 0,3596 \\
\hline$x_{3}(R)$ & 42,0984 & 40,3239 & 48,3290 & 40,4606 & 38,3211 & 37,6990 \\
\hline$x_{4}(L)$ & 176,6366 & 200 & 112,6790 & 198,1174 & 229,8254 & 239,9999 \\
\hline$g_{1}$ & 0 & $-0,0343$ & $-0,0047$ & $-0,0001$ & $0,15 \mathrm{E}-5$ & $3,91 \mathrm{E}-5$ \\
\hline$g_{2}$ & $-0,0358$ & $-0,0528$ & $-0,0389$ & $-0,0001$ & $-0,0006$ & $1,26 \mathrm{E}-5$ \\
\hline$g_{3}$ & 0 & $-27,1058$ & $-3652,7888$ & $-370,3341$ & $-15,105$ & $3,25 \mathrm{E}-9$ \\
\hline$g_{4}$ & $-63,3624$ & -40 & $-127,3210$ & $-41,8825$ & $-10,1745$ & $1,54 \mathrm{E}-18$ \\
\hline$f$ & 6059,1312 & 6288,7445 & 6410,3811 & 5892,8890 & 5824,2572 & 5804,2071 \\
\hline NAFO & - & - & - & 121100 & 100100 & 65228 \\
\hline
\end{tabular}

Os resultados apresentados nesta tabela demonstram que os algoritmos ACV e ACVA foram os que obtiveram os melhores valores em comparação com os resultados reportados pela literatura. Todavia, em relação ao Algoritmo Line-up e ao ACV tradicional, o ACVA conseguiu reduzir em cerca de, aproximadamente, 46,14\% e 34,84\% o número de avaliações da função objetivo.

A Figura 4 apresenta a variação do valor da função objetivo, da taxa de convergência, do tamanho da população e dos parâmetros do ACVA.

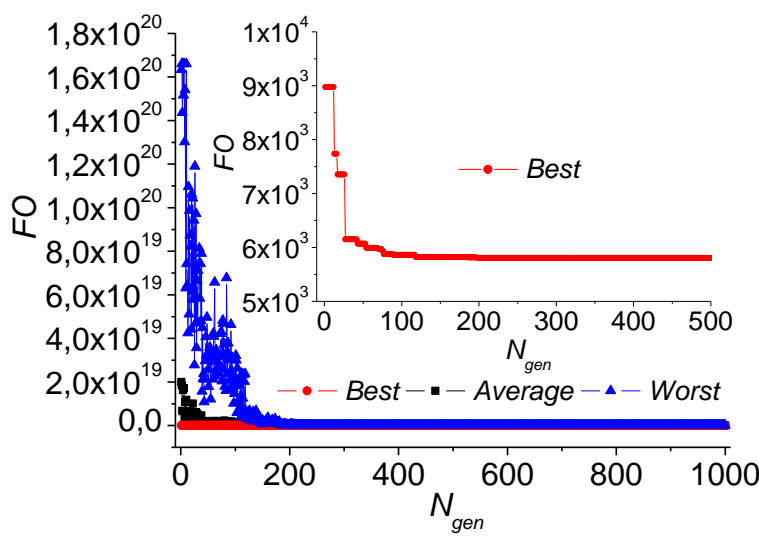

(a) Função Objetivo.

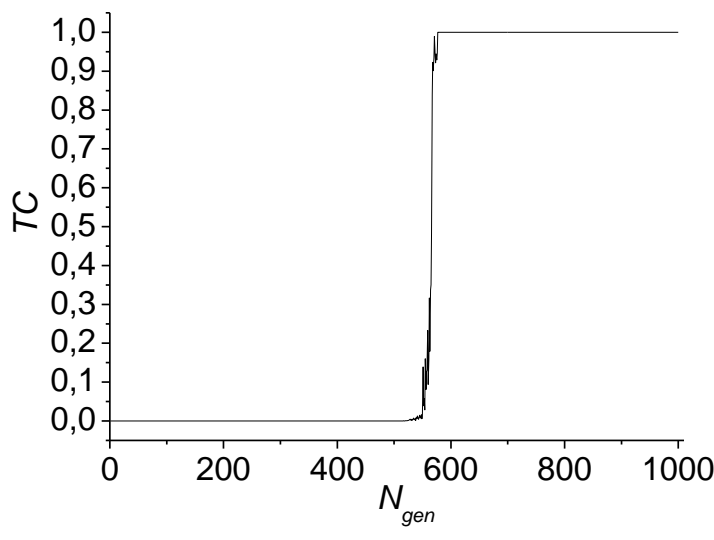

(b) Taxa de Convergência. 


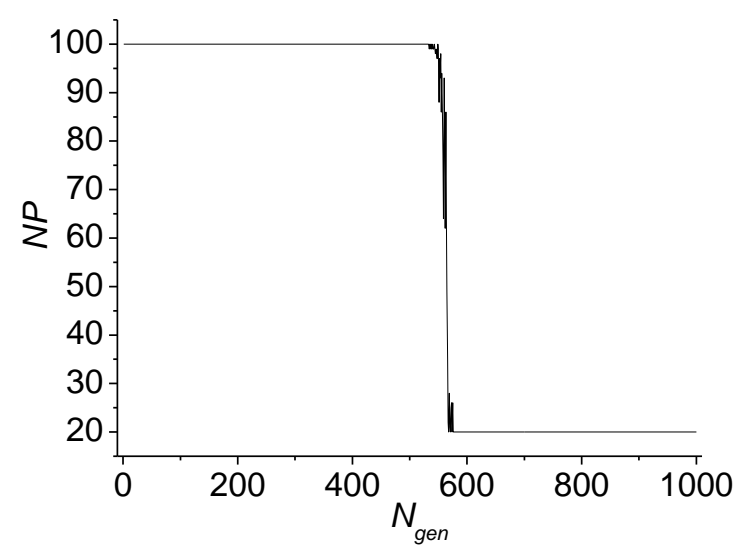

(c) Tamanho da População.

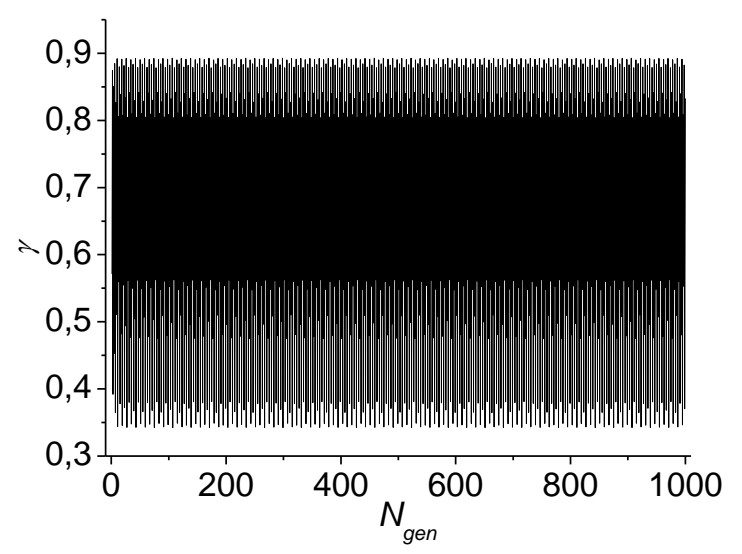

(e) Parâmetro de Absorção da Luz.

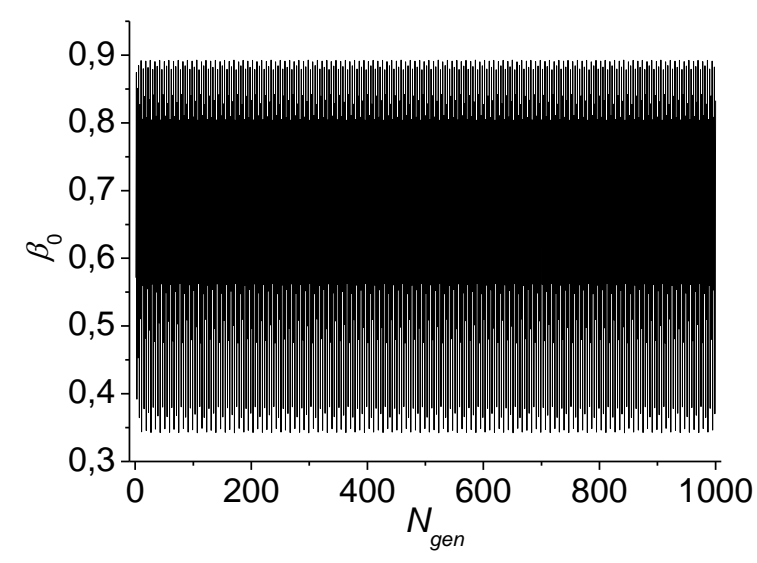

(d) Fator de Atratividade.

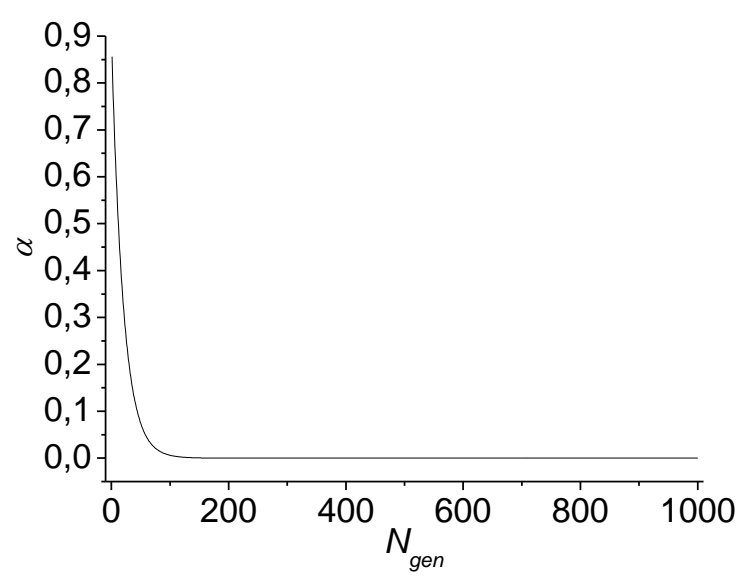

(f) Parâmetro de Inserção de Regularidade.

Figura 4. Evolução da função objetivo e dos parâmetros do ACVA para o problema do projeto de um recipiente de pressão.

Conforme comentado anteriormente, na Figura 4(a) observa-se que ao longo do processo evolutivo a população tende a ser tornar homogênea, implicando no aumento do valor da taxa de convergência e posterior redução do tamanho da população, conforme observado nas Figuras 4(b) e 4(c). Já nas Figuras 4(d), 4(e) e 4(f), observa-se a forma pela qual os parâmetros do ACVA é atualizada em uma dada corrida.

O último estudo de caso trata do projeto de uma viga engastada cujo objetivo é minimizar o custo de uma viga (Equação (27)) com as limitações de tensão de cisalhamento (Equação (28)), tensão de dobramento na viga (Equação (29)), esforço de carga na barra (Equação (30)), reflexão final da viga (Equação (31)) e restrições laterais (Equações (32) e (33)), conforme expresso na Figura 5 (Coello, 2000; Deb, 1997). 


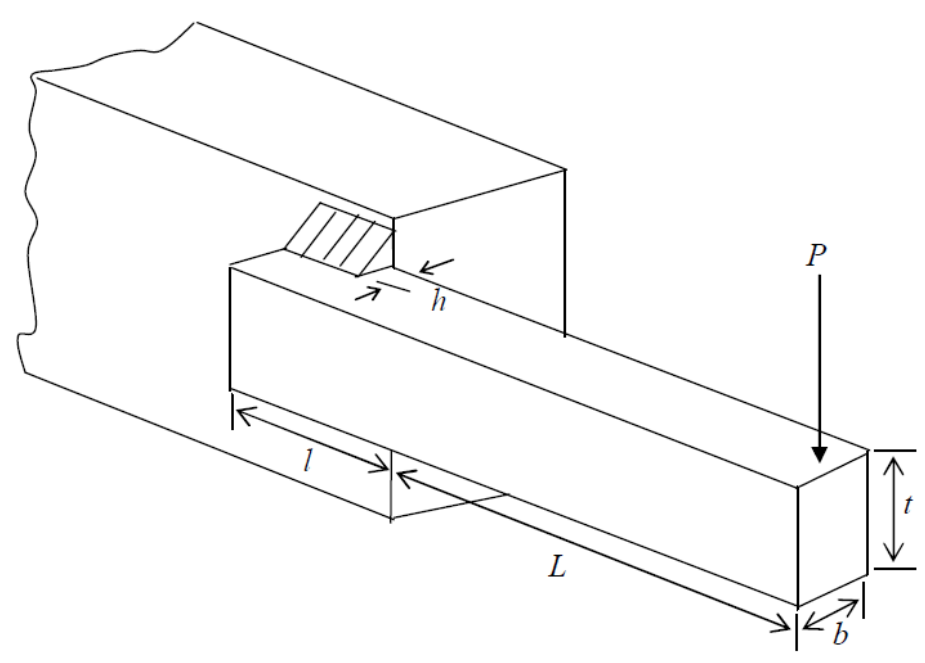

Figura 5. Projeto de uma viga engastada.

Matematicamente, esse problema pode ser descrito conforme segue:

$$
\min f=1,10471 x_{1}^{2} x_{2}+0,04811 x_{3} x_{4}\left(14+x_{2}\right)
$$

sujeito a

$$
\begin{gathered}
g_{1} \equiv \sqrt{\left(\frac{P}{\sqrt{2} x_{1} x_{2}}\right)^{2}+2\left(\frac{P}{\sqrt{2} x_{1} x_{2}}\right)\left(\frac{M R}{J}\right) \frac{x_{2}}{2 R}+\left(\frac{M R}{J}\right)^{2}}-\tau_{\max } \leq 0 \\
g_{2} \equiv \frac{6 P L}{x_{4} x_{3}^{2}}-\sigma_{\max } \leq 0 \\
g_{3} \equiv x_{1}-x_{4} \leq 0 \\
g_{4} \equiv 0,10471 x_{1}^{2}+0,04811 x_{3} x_{4}\left(14+x_{2}\right)-5 \leq 0 \\
g_{5} \equiv 0,125-x_{1} \leq 0 \\
g_{6} \equiv \frac{4 P L^{3}}{E x_{3}^{3} x_{4}}-\delta_{\max } \leq 0 \\
g_{7} \equiv P-P_{C} \leq 0 \\
0,1 \leq x_{1} \leq 2, \quad 0,1 \leq x_{2} \leq 10, \quad 0,1 \leq x_{3} \leq 10, \quad 0,1 \leq x_{4} \leq 2
\end{gathered}
$$

com

$$
M \equiv P\left(L+\frac{x_{2}}{2}\right)
$$




$$
\begin{gathered}
R \equiv \sqrt{\frac{x_{2}^{2}}{4}+\left(\frac{x_{1}+x_{3}}{2}\right)^{2}} \\
J \equiv 2\left(\sqrt{2} x_{1} x_{2}\left(\frac{x_{2}^{2}}{12}+\left(\frac{x_{1}+x_{3}}{2}\right)^{2}\right)\right) \\
P_{C} \equiv \frac{4,013 E}{L^{2}} \sqrt{\frac{x_{3}^{2} x_{4}^{6}}{36}}\left(1-\frac{x_{3}}{2 L} \sqrt{\frac{E}{4 G}}\right)
\end{gathered}
$$

e $P=6000 \mathrm{lb}, L=14 \mathrm{in}, E=30 \times 10^{6} \mathrm{psi}, G=12.10^{6} \mathrm{psi}, T_{\max }=13600 \mathrm{psi}, \sigma_{\max }=30000 \mathrm{psi}$ e $\delta_{\max }=0,25 \mathrm{in}$.

Para resolver este estudo de caso, os seguintes parâmetros foram considerados: ACV $\left(N P=100, N_{g e n}=1000, \alpha=0,9 ; \beta_{0}=0,8\right.$ e $\left.\gamma=0,8\right) ;$ ACVA $\left(N P_{\min }=20, N P_{\max }=100, N_{g e n}=1000\right.$, e os parâmetros $\alpha, \beta_{0}$ e $\gamma$ sendo atualizados conforme descrito anteriormente) e o Método da Penalização Estática (com parâmetro de penalidade da ordem de $10^{8}$ ).

A Tabela 3 apresenta os resultados obtidos pelos algoritmos ACV e ACVA em comparação com os resultados descritos na literatura considerando outras estratégias evolutivas.

Tabela 3. Resultados obtidos com os algoritmos ACV e ACVA.

\begin{tabular}{ccccccc}
\hline & Hu et al. (2003) & Coello (2000) & Deb (1997) & Masta et al. (2012) & ACV & ACVA \\
\hline$x_{1}(h)$ & 0,2057 & 0,2088 & 0,2489 & 0,2056 & 0,2045 & 0,2057 \\
\hline$x_{2}(h)$ & 3,4704 & 3,4205 & 6,1730 & 3,4780 & 3,4987 & 3,4704 \\
\hline$x_{3}(t)$ & 9,0366 & 8,9975 & 8,1739 & 9,0356 & 9,0344 & 9,0366 \\
\hline$x_{4}(b)$ & 0,2057 & 0,2100 & 0,2533 & 0,2045 & 0,2058 & 0,2057 \\
\hline$g_{1}$ & 0,0000 & $-0,3378$ & $-5758,6033$ & 0,0000 & $-5,0738$ & $6,91 \mathrm{E}-11$ \\
\hline$g_{2}$ & 0,0000 & $-353,9026$ & $-255,5760$ & 0,0000 & $-7,5110$ & $6,84 \mathrm{E}-11$ \\
\hline$g_{3}$ & 0,0000 & $-0,0012$ & $-0,0044$ & 0,0000 & $-0,0013$ & $3,38 \mathrm{E}-6$ \\
\hline$g_{4}$ & $-3,4329$ & $-3,4118$ & $-2,9828$ & $-3,4389$ & $-3,4297$ & $-3,4329$ \\
\hline$g_{5}$ & $-0,0800$ & $-0,0838$ & $-0,1239$ & $-0,0300$ & $-0,0795$ & $-0,0807$ \\
\hline$g_{6}$ & $-0,2355$ & $-0,2356$ & $-0,2342$ & $-0,2222$ & $-0,2355$ & $-0,2355$ \\
\hline$g_{7}$ & 0,0000 & $-363,2323$ & $-4465,2709$ & 0,0000 & $-12,1157$ & $1,18 \mathrm{E}-10$ \\
\hline$f$ & 1,7248 & 1,7483 & 2,4331 & 1,7214 & 1,7215 & 1,7216 \\
\hline NAFO & - & - & - & 111100 & 100100 & 79285 \\
\hline
\end{tabular}

Assim como no estudo de caso anterior, os resultados obtidos demonstram a habilidade dos algoritmos ACV e ACVA em comparação com outras estratégias evolutivas. Em relação ao Algoritmo Line-up e ao ACV tradicional, o ACVA conseguiu reduzir em cerca de, aproximadamente, 28,64\% e 20,80\% o número de avaliações da função objetivo. Já a Figura 6 apresenta a variação do valor da função objetivo, da taxa de convergência, do tamanho da população e dos parâmetros do ACVA. 


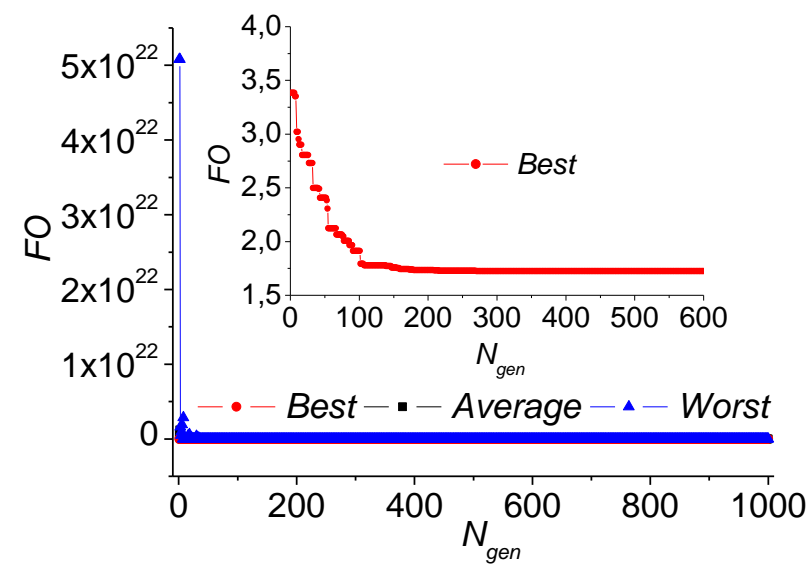

(a) Função Objetivo.

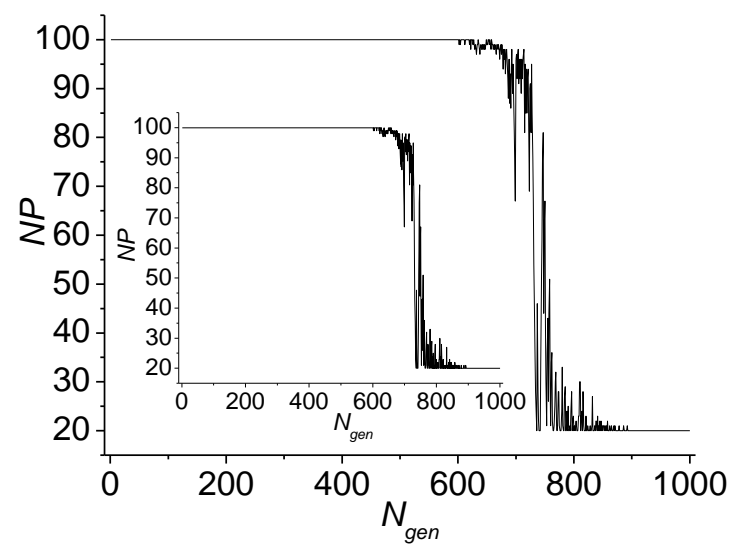

(c) Tamanho da População.

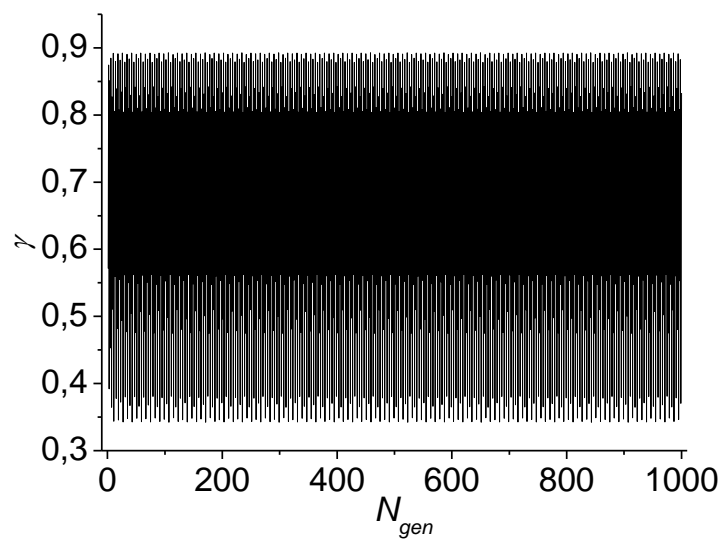

(e) Parâmetro de absorção da luz.

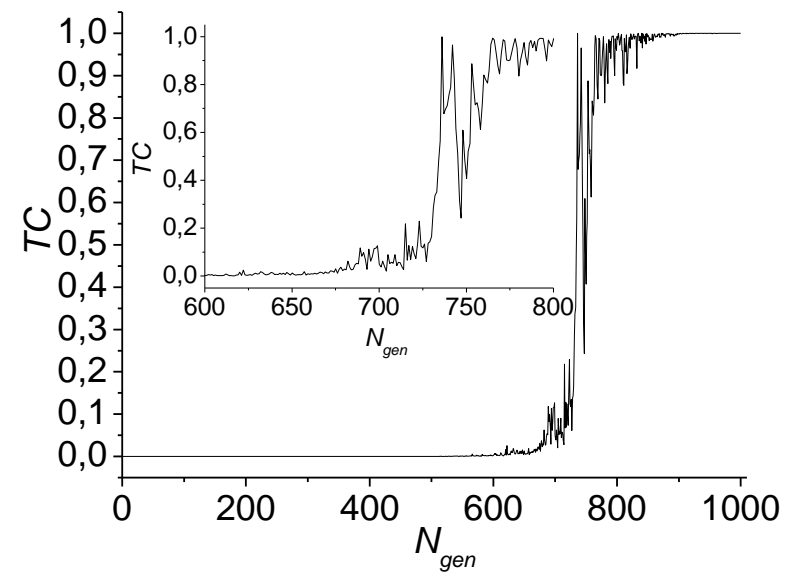

(b) Taxa de Convergência.

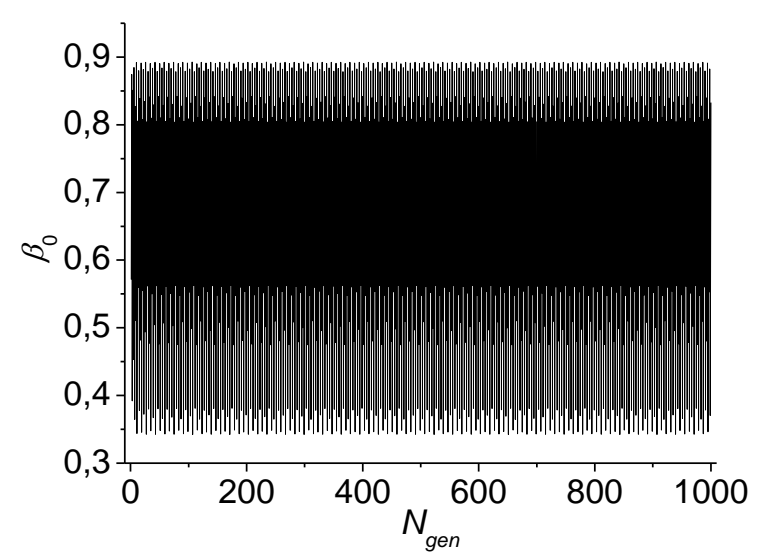

(d) Fator de atratividade.

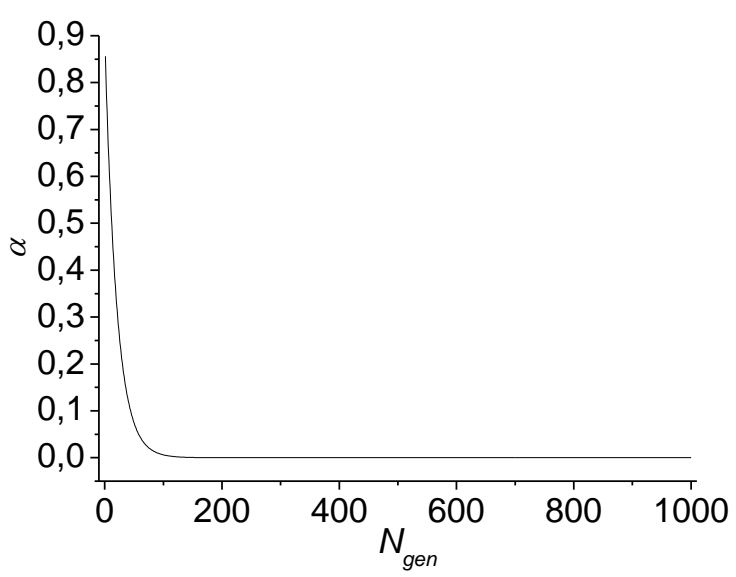

(f) Parâmetro de Inserção de Regularidade.

Figura 6. Evolução da função objetivo e dos parâmetros do ACVA para o problema do projeto de uma viga engastada. 
Na Figura 6(a) observa-se que a população tende a ser tornar homogênea ao longo do processo evolutivo, o que influencia no perfil da taxa de convergência e, consequentemente, no perfil referente ao tamanho da população, conforme observado nas Figuras 6(b) e 6(c). Já nas Figuras 6(d), 6(e) e 6(f), observa-se a forma pela qual os parâmetros do ACVA é atualizada em uma dada corrida.

\section{CONCLUSOES}

Este trabalho apresentou uma estratégia evolutiva auto-adaptativa baseada no Algoritmo de Colônia de Vagalumes associado a uma estratégia para atualização do tamanho da população baseada no conceito de taxa de convergência, a uma estratégia baseada em um Modelo Caótico de Busca para a atualização do fator de atratividade e do parâmetro de absorção da luz pelo meio, de uma relação empírica para a atualização do parâmetro de inserção de regularidade, e do conceito de sub-populações para a inserção de diversidade ao processo de busca pela solução global.

A partir da análise dos resultados obtidos com a aplicação da metodologia proposta em funções matemáticas e com o projeto de dois sistemas de engenharia com diferentes níveis de complexidade, foi possível observar que a abordagem proposta sempre foi capaz de obter uma solução factível com a reportada pela literatura. Todavia, ressalta-se que às custas de um número menor de avaliações da função objetivo em relação à diferentes versões do ACV e outras estratégias evolutivas.

Como sequencia deste trabalho pretende-se propor a utilização de outros modelos caóticos de busca, bem como a extensão da metodologia proposta para o contexto multiobjetivo.

\section{AGRADECIMENTOS}

Os autores agradecem ao CNPq e a FAPEMIG pelo suporte financeiro necessário para a realização deste trabalho. Carvalho agradece a CAPES por sua bolsa de pósgraduação.

\section{REFERENCIAS}

ADJIMAN, C. S.; SALLWIG, S.; FLOUDA, C. A.; NEUMAIER, A. A Global Optimization Method for General Twice-Differentiable NLPs-1 - Theoretical Advances. Computers Chemical Engineering, 22, 9, 1137-1158, 1998. 
ABSHOURI, A. A.; MEYBODI, M. R.; BAKHTIARY, A. New Firefly Algorithm Based on Multiswarm and Learning Automata in Dynamic Environments. Third Int. Conference on Signal Processing Systems (ICSPS2011), Aug 27-28, Yantai, China, pp. 73-77 (2011).

ALI, M. M.; TORN, A. Population Set Based Global Optimization Algorithms: Some Modifications and Numerical Studies. Computers and Operations Research, 31, 17031725, 2004.

AYDILEK, I. B. A Hybrid Firefly and Particle Swarm Optimization Algorithm for Computationally Expensive Numerical Problems. Applied Soft Computing, 66, 232249, 2018.

AZAD, S. K.; AZAD, S. K. Optimum Design of Structures Using an Improved Firefly Algorithm. International Journal of Optimisation in Civil Engineering, 1(2), 327-340, 2011.

BASU, B.; MAHANTI, G. K. Firefly and Artificial Bees Colony Algorithm for Synthesis of Scanned and Broadside Linear Array Antenna. Progress in Electromagnetic Research B., 32, 169-190 2011.

CHATTERJEE, A.; MAHANTI, G. K.; CHATTERJEE, A. Design of a Fully Digital Controlled Reconfigurable Switched Beam Conconcentric Ring Array Antenna using Firefly and Particle Swarm Optimisation Algorithm. Progress in Elelectromagnetic Research B., 36, 113-131, 2012.

COELLO, C. A. C. Use of a Self-adaptive Penalty Approach for Engineering Optimization Problems, Computers in Industry, 41 (2), 113-127, 2000.

CSENDES,T.; RATZ, D. Subdivision-Direction Selection in Interval Methods for Global Optimization. SIAM Journal on Numerical Analysis, 34, 3, 922-938, 1997.

DAS, T. K.; VENAYAGAMOORTHY, G.K. Bio-inspired Algorithms for the Design of Multiple Optimal Power System Stabilizers: SPPSO and BFA. Industry Applications Conference, 41 IAS Annual Meeting. Conference Record of the 2006 IEEE, 2, 635-641, 2006.

DEB, K. GenaAS: A Robust Optimal Design Technique for Mechanical Component Design, Dasgupta, D. and Michalewicz 2 (Eds). Evolutionary Algorithms in Engineering Applications, Berlin, pp. 497-514, 1997.

DEVANEI, R. L. An Introduction to Chaotic Dynamical Systems. Addison-Wesley, 1987.

FARAHANI, S. M.; ABSHOURI, A. A.; NASIRI, B.; MEYBODI, M. R. A Gaussian Firefly Algorithm. Int. J. Machine Learning and Computing, 1(5), 448-453, 2011.

FARAHANI, S. M.; NASIRI, B.; MEYBODI, M. R. A Multiswarm Based Firefly Algorithm in Dynamic Environments. Third Int. Conference on Signal Processing Systems (ICSPS2011), Aug 27-28, Yantai, China, 68-72, 2011.

HORNG, M.-H.; LEE, Y.-X.; LEE, M.-C.; LIOU, R.-J. Firefly Metaheuristic Algorithm for Training the Radial Basis Function Network for Data Classification and Disease Diagnosis. Theory and New Applications of Swarm Intelligence (Edited by R. Parpinelli and H. S. Lopes), 115-132, 2012. 
HU, X.; EBERHART, R.; SHI, Y. Engineering Optimization with Particle Swarm, IEEE Conference on Swarm Intelligence, Indianapolis-EUA, 2003.

KENNEDY, J.; EBERHART, R. Particle Swarm Optimization. In Proceedings of IEEE International Conference on Neural Networks, 1942-1948, 1995.

LEIDENFROST, R.; ELMENREICH, W. Establishing Wireless Time-Triggered Communication using a Firefly Clock Synchronization Approach. Proceedings of the 2008 International Workshop on Intelligent Solutions in Embedded Systems, 1-18, 2008.

LI, B.; JIANG, W. Optimization of Complex Functions by Chaos Search. International Journal of Cybernetics and Systems, 29 (4), 409-419, 1998.

LIEU, Q. X.; DO, D. T. T.; Lee, J. An Adaptive Hybrid Evolutionary Firefly Algorithm for Shape and Size Optimization of Truss Structures with Frequency Constraints. Computers \& Structures, 195, 99-112, 2018.

LUZ, E. F. P.; BECCENERI, J. C.; CAMPOS VELHO, H. F. Conceitualização do Algoritmo Vagalume e sua Aplicação na Estimativa de Condição Inicial da Equação de Calor. IX Workshop do Curso de Computação Aplicada do INPE (WORCAP 2009), São José dos Campos, 2009.

MASTA, I. B.; LOBATO, F. S.; MALAGONI, R. A.; VIERIA, P. A. Projeto de Sistemas de Engenharia Mecânica usando o Algoritmo Line-Up. Simpósio de Mecânica Computacional, 23 a 25 de Maio na Escola de Engenharia da Universidade Federal de Minas Gerais, 2012.

OTT, E. Chaos in Dynamical Systems. Cambridge University Press, Cambridge, UK, 2002.

RAHNAMYAN, S.; TIZHOOSH, H. R.; SALAMA, N. M. M. A Novel Population Initialization Method for Accelerating Evolutionary Algorithms. Computers and Mathematics with Applications, 53, 10, 1605-1614, 2007a.

RAHNAMYAN, S.; TIZHOOSH, H. R.; SALAMA, N. M. M. Opposition-Based Differential Evolution (ODE) with Variable Jumping Rate. IEEE Symposium Foundations Computation Intelligence, Honolulu, HI, pp.81-88, 2007b.

SARIMVEIS, H.; NIKOLAKOPOULOS, A. A Line Up Evolutionary Algorithm for Solving Nonlinear Constrained Optimization Problems. Computers \& Operations Research, 32, 1499-1514, 2005.

SAYADI, M. K.; RAMEZANIAN, R.; GHAFFARI-NASAB, N. A Discrete Firefly MetaHeuristic with Local Search for Makespan Minimization in Permutation Flow Shop Scheduling Problems. Int. J. of Industrial Engineering Computations, 1, 1-10, 2010.

SENTHILNATH, J.; OMKAR, S. N.; MANI, V. Clustering using Firely Algorithm: Performance Study. Swarm and Evolutionary Computation, 1(3), 164-171, 2011.

TAVAZOEI, M. S.; HAERI, M. Comparison of Different One-Dimensional Maps as Chaotic Search Pattern in Chaos Optimization Algorithms. Applied Mathematics and Computation, 187, 1076-1085, 2007. 
Algorithm for Global Optimization. European Journal of Operational Research, 183, 5673, 2007.

WANG, H.; WANG, W.; ZHOU, X.; SUN, H.; ZHAO, J.; YU, X.; CUI, Z. Firefly Algorithm with Neighborhood Attraction. Information Sciences, 382, 374-387, 2017.

WERNER-ALLEN, G.; TEWARI, G.; PATEL, A.; WELSH, M.; NAGPAL, R. FireflyInspired Sensor Network Synchronicity with Realistic Radio Effects. Proceedings of the Third International Conference on Embedded Networked Sensor Systems, 142-153, 2005.

YANG, X.-S. Nature-Inspired Metaheuristic Algorithms. Luniver Press, Cambridge, 2008.

YANG, D.; LI, D.; CHENG, G. On the Efficiency of Chaos Optimization Algorithms for Global Optimization. Chaos, Solutions and Fractals, 34, 1366-1375, 2007.

YANG, X.-S. Test Problems in Optimization. Engineering Optimization: An Introduction with Metaheuristic Applications, John Wiley \& Sons [online] http://arxiv.org/abs/1008.0549, 2010. 